\title{
La necesidad de renovar el sistema de atención de salud mental desde un enfoque integral
}

\section{Giovani Díaz-Gervasi ${ }^{1}$ \\ Yordanis Enríquez-Canto ${ }^{2}$}

\author{
${ }^{1}$ Universidad Católica Sedes Sapientiae. \\ Facultad de Ciencias de la Salud. \\ Magíster en Psicología clínica.

\begin{abstract}
${ }^{2}$ Universidad Católica Sedes Sapientiae. Facultad de Ciencias de la Salud.
\end{abstract} \\ Doctor en Bioética.
}

Recibido: 03-04-2017 Aprobado: 15-04-2017

Citar como: Díaz G, Enríquez Y. La necesidad de renovar el sistema de atención de salud mental desde un enfoque integral. CASUS. 2017;2(1):7-10.
La visión actual de la salud tiende cada vez más a considerar las necesidades de la persona no solo en lo físico o mental, sino también los diversos aspectos psicosociales y del entorno en que vive (1). Esto no es mera consecuencia de un planteamiento teórico, sino de la experiencia que solicita cada vez más una concepción amplia de la atención en salud. A medida que nuestra comprensión de la salud y de la enfermedad mental continúa evolucionando, se hacen indispensables nuevas propuestas en el sistema de prestación de servicios de salud mental $(1,2)$.

A nivel nacional al año 2015 las formas más comunes de enfermedad mental fueron el trastorno depresivo (20.7\%), la violencia (11.3\%) y las adicciones (3\%) (3). Otras enfermedades como la ansiedad, los trastornos de la alimentación, el consumo de sustancias, sobre todo de alcohol se han convertido en temas recurrentes de conversación, ya que las personas en estas circunstancias manifiestan su dificultad para obtener ayuda. Sin embargo, solo un $20 \%$ del total de los afectados por enfermedades mentales buscan ayuda especializada (3). Esto se debe en gran parte al estigma que rodea la enfermedad mental (1). Igualmente, a las personas que buscan ayuda, el actual sistema de salud mental les suele parecer poco accesible y caracterizado por servicios fragmentados. Esto puede generar sentimientos de frustración frente al proveedor de salud ya que fracasa la esperanza de recibir el tratamiento necesario para la recuperación $(1,2)$. Al igual que el abordaje terapéutico de cualquier enfermedad, el tratamiento eficaz de las enfermedades mentales y del comportamiento requiere tanto la continuidad de la atención, como la combinación adecuada de servicios cuándo y dónde la persona los necesite (1).

En nuestro contexto se vienen haciendo esfuerzos para mejorar el acceso a servicios especializados de salud mental sobre todo para los que están más afectados. El Ministerio de Salud logró la modificación del artículo 11 de la Ley 26842, Ley General de Salud. La misma garantiza los derechos de las personas con problemas de salud mental sin diferenciación. El artículo 11 refiere que todas las personas tienen derecho a gozar de salud mental y que es responsabilidad del Estado garantizar la disponibilidad de programas en todo el territorio nacional. En 
esta línea también se consideran como acciones básicas la promoción, prevención, recuperación y rehabilitación.

En este sentido, la Dirección de Salud Mental en el 2015 inauguró 22 Centros de Salud Mental Comunitario en el territorio nacional (3). Esta implementación sigue los nuevos métodos de tratamiento a nivel nacional e internacional. Los cuales van más allá de la atención tradicional, circunscrita al consultorio y al ingreso a áreas de salud mental en un hospital $(1,2)$. Sin embargo, en nuestro contexto, de acuerdo a la demanda de atención de salud mental estos servicios resultan aún insuficientes. En cambio, estas iniciativas con la finalidad de tener una mayor efectividad y alcance deben también considerar los aspectos sociales de la salud mental.

Una visión integral de salud incluye también los determinantes sociales de la misma, como el nivel socioeconómico, la educación y las redes de apoyo social que influyen significativamente en la salud mental (4). El nivel de ingresos es uno de los determinantes sociales más significativos, ya que influye en la calidad de otros factores determinantes como la vivienda, la seguridad alimentaria, la educación y la actividad física (4).

Existe fuerte evidencia de que una mejor situación social y económica está asociada a mayores índices de salud (4). A medida que aumenta el ingreso las necesidades de atención médica generalmente disminuyen (4). La falta de vivienda, la pobreza, el desempleo y la baja alfabetización exponen a las personas a un riesgo mayor de desarrollar problemas de salud mental (4). Del mismo modo, estos problemas pueden influir negativamente en la capacidad de un individuo para encontrar una vivienda segura y estable, obtener empleo e ingresos adecuados (4). Tomar medidas para mejorar los determinantes sociales es esencial para promover y mantener la salud mental $(1,2)$.

Considerando lo anterior se comprende la urgencia de prestar mayor atención a un sistema de salud que considere a la comunidad y sus necesidades (5) haciendo especial hincapié en la promoción y prevención de la salud mental (1). A través de un modelo integrado de atención primaria de salud que comprenda el tratamiento, la recuperación y el apoyo entre pares $(1,2,5)$. Desde este punto de vista la intervención temprana es esencial $(1,5)$.

Si bien se reconoce que es necesario algún tipo de infraestructura y de servicios de hospitalización, sin embargo esto no justifica que se ubique a todos los necesitados en hospitales psiquiátricos $(1,5)$. El Ministerio de Salud debe indagar en las opciones para reemplazar el hospital con otros servicios de hospitalización alternativos, así como en más servicios prestados en la comunidad.

La transformación del sistema de salud mental en el Perú requerirá un esfuerzo conjunto y combinado de todo el Gobierno, trabajando en 
estrecha colaboración con las autoridades sanitarias regionales, los proveedores de atención médica, las personas con vivencia de la enfermedad mental, sus familias y los comités comunitarios $(1,2,5)$. Una nueva visión para un sistema de salud mental se focalizaría en los siguientes aspectos: mejor acceso a más servicios; mejor calidad de la atención; mejor programación de actividades de promoción, prevención, atención, seguimiento y reinserción; además de fortalecer el apoyo y la organización de la comunidad (5).

Otros aspectos fundamentales de esta visión son el empoderar a las personas alentándolas a ser partícipes de la planificación de su cuidado $(2,5)$. Por ello, los programas y servicios centrados en la recuperación deben inspirar esperanza y capacitar en la búsqueda de la salud mental y del bienestar (1).

Bajo este enfoque se debería proporcionar a las familias mayor acceso a programas $(1,2)$ que se centren en el desarrollo de habilidades de afrontamiento de los padres. Los cuales tendrían la finalidad de incrementar la resiliencia, desarrollar las habilidades de crianza de los hijos y reforzar las competencias sociales y emocionales en los niños. Además de brindar un apoyo sistemático a familias y cuidadores de personas que viven con enfermedades mentales y del comportamiento $(1,5)$.

Asimismo, en la escuela se requeriría implementar un marco integral de salud y bienestar $(2,5)$. El cual figurando en el currículo de cada grado puede incluir programas de prevención e integración de la salud mental (2). Además ayudar a los estudiantes a identificar y manejar eficazmente el estrés y la ansiedad, el estilo asertivo de comunicación, el reconocimiento emocional y sus capacidades cognitivas. Mientras que por parte del Estado se pueden crear equipos regionales interdisciplinarios permanentes para preservar e intervenir en la salud mental de los escolares $(1,5)$. La creación de los mismos requiere un replanteamiento de las funciones de los psicólogos educativos, trabajadores sociales y maestros para determinar la mejor manera de responder a las necesidades de salud mental en el ambiente escolar y los recursos necesarios para hacerlo $(1,2)$.

A través de este enfoque se deben desarrollar y ofrecer una gama de servicios de salud mental integrados dentro de los servicios comunitarios y de atención primaria existentes $(1,2,5)$. Los cuales incluirían programas de autogestión para el bienestar mental y programas de fortalecimiento familiar para niños, jóvenes y sus familias (2). Igualmente a nivel psicológico se pueden ofrecer psicoterapias desarrolladas por terapeutas in situ o en línea para el manejo de la depresión, la ansiedad y las adicciones.

Otros servicios de salud mental (2) pueden incluir programas de tratamiento diurno, acceso a servicios de internamiento, clínicas temporáneas ambulatorias, equipos de tratamiento y entrenamiento asertivo en la comunidad, así como equipos móviles de intervención en crisis (5). Existe evidencia de que los servicios prestados a la 


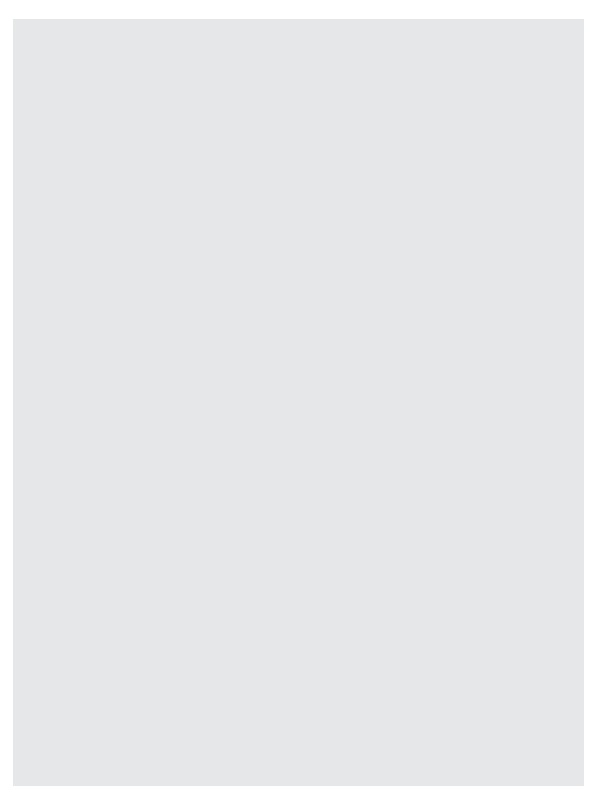

comunidad poseen aceptabilidad, resultan eficientes y conducen a una mejor calidad de vida y a menores tasas de hospitalización $(1,2)$.

Responder a los problemas de salud mental es una responsabilidad compartida y requiere el apoyo del Gobierno, del sector salud y de la comunidad. Los sectores gubernamentales, las autoridades sanitarias regionales, las comunidades y las personas con la experiencia de la enfermedad mental deben trabajar juntos para abordar estos desafíos. Los mismos deben eliminar las barreras $(1,2)$, centrarse en las personas $(1,2)$ y aprender nuevas formas conjuntas de trabajar.

Un nuevo sistema de atención de la salud mental promueve el bienestar mental, la resiliencia y apoya a las personas y familias con la vivencia de la enfermedad mental a vivir en modo gratificante una vida plena $(1,2)$. Necesitamos un sistema integrado centrado en la persona y en la prevención temprana. La transformación del sistema es una alta prioridad para la población peruana y requiere de acciones inmediatas.

\section{REFERENCIAS BIBLIOGRÁFICAS}

1. Collins P. et al. Grand challenges in global mental health. Nature. 2011;475(7354):27-30.

2. Fieldhouse J. Community Participation and Recovery for Mental Health Service Users: An Action Research Inquiry. British Journal of Occupational Therapy. 2012;75(9):419-428.
3. MINSA. Memoria Anual 2015. Lima: MINSA; 2015.

Rocha K, Pérez K, Rodríguez M,

4. Borrell C, Obiols J. Prevalencia de problemas de salud mental y su asociación con variables socioeconómicas, de trabajo y salud: resultados de la Encuesta Nacional de Salud de España. Psicothema. 2010;22(3):389-395.
5. Ardila S, Galende E. El concepto de comunidad en la salud mental comunitaria. Salud Mental y Comunidad. 2011;1(1):39-50. 